\title{
Estimation of Plaque Contents With Multi-Angle 3D Compound Imaging
}

Wilhjelm, Jens E.; Grønholdt, Marie Louise; Rasmussen, Steen Tofthøj; Martinsen, Kjeld; Sillesen, Henrik

\section{Published in:}

Proceedings of the IEEE International Ultrasonics Symposium

Link to article, DOI:

10.1109/ULTSYM.1996.584178

Publication date:

1996

Document Version

Publisher's PDF, also known as Version of record

Link back to DTU Orbit

Citation (APA):

Wilhjelm, J. E., Grønholdt, M. L., Rasmussen, S. T., Martinsen, K., \& Sillesen, H. (1996). Estimation of Plaque Contents With Multi-Angle 3D Compound Imaging. In Proceedings of the IEEE International Ultrasonics Symposium (pp. 1077-1080). IEEE. https://doi.org/10.1109/ULTSYM.1996.584178

\section{General rights}

Copyright and moral rights for the publications made accessible in the public portal are retained by the authors and/or other copyright owners and it is a condition of accessing publications that users recognise and abide by the legal requirements associated with these rights.

- Users may download and print one copy of any publication from the public portal for the purpose of private study or research.

- You may not further distribute the material or use it for any profit-making activity or commercial gain

- You may freely distribute the URL identifying the publication in the public portal 


\title{
Estimation of Plaque Contents With Multi-Angle 3D Compound Imaging
}

\author{
J. E. WILHJELM ${ }^{1}$, M.-L. M. GRøNHOLDT ${ }^{2}$, S. RASMUSSEN ${ }^{1}$, K. MARTINSEN ${ }^{1}$ \& H. SILLESEN ${ }^{2}$ \\ Center for Arteriosclerosis Detection with Ultrasound (CADUS) \\ E-mail: wilhjelm@it.dtu.dk. Homepage: http:/www.it.dtu.dk/ wilhjelm/cadus.html \\ ${ }^{1}$ Dept. of Information Tech., Tech. Univ. of Denmark, Bldg. 344, DK-2800 Lyngby, Denmark. \\ ${ }^{2}$ Dept. of Vascular Surgery, Rigshospitalet, University of Copenhagen, \\ Blegdamsvej 9, DK-2100 Copenhagen $\varnothing$, Denmark.
}

\begin{abstract}
This investigation exploits the potential of using multiple insonification angles in characterizing plaques in the carotid artery. Specifically, previous work has shown that certain plaque materials such as lipid exhibit a low degree of angle-dependence in the received echo signal while the signal varies much more with angle for fibrous tissues.

An experimental multi-angle $3 D$ pulse-echo recording system has been established with a $10 \mathrm{MHz}$ focused single element transducer mounted on a high precision rotational device. The received signal was processed in order to match axial resolution size to lateral resolution size (1.4 mm@-3 dB) to obtain an omnidirectional range cell. After scanning, the plaques underwent histological analysis in order to estimate the amount of calcification, fibrous tissues, lipid, blood and thrombus.

So far, 13 plaques surgically removed during carotid endarterectomy have been scanned in $3 D$ from five different angles. Based on the angle-dependence of the signal energy for each range cell, six features could be extracted and correlated to the histological results. From the limited amount of data presented here, correlation was found between one feature and the content of fibrous tissues. The preliminary results indicate that the method has good potential for characterization of plaque.
\end{abstract}

\section{Introduction}

The classification of carotid artery plaques remains a challenge for all current non-invasive imaging techniques. An unstable carotid plaque may lead to formation of emboli which in turn might cause cerebral infarction (e.g. stroke). Reduction of this risk is possible with carotid endarterectomy, i.e. the plaque is removed surgically. ${ }^{[3,5]}$ For the purpose of selecting patients for surgery, recent research suggests that plaque composition and plaque shape are important factors in plaque instability. ${ }^{[1.2]}$ If these factors can be estimated non-invasively, an additional tool for selection of patients for operation would be available.

Ultrasonic investigation of plaques in vitro has been underway for many years. In 1985, Picano et al. ${ }^{[6]}$ published evidence of distinct differences in the received ultrasound signals from aortic plaques of different types Specifically, for plaques with a high content of lipid materials, the signal amplitude was low and rather constant with changing angle of insonification. In contrast, highly calcified plaques produced signals of very high magnitude, with a significant angle-dependence. For plaques consisting mainly of fibrous materials, the behavior was in between the two groups mentioned above.

This paper reports on the initial findings of a larger study aimed at mapping plaque material content in the entire 3D plaque. Specifically, data were obtained by scanning plaques in vitro (removed after carotid endarterectomy) in 3D from five different angles. The data were subsequently correlated with the results of an histological examination of the plaques.

\section{Ultrasound System}

The experimental ultrasound system consisted of a pulser/receiver (type 5072 PR, Panametrics, Waltham, MA, USA) connected to a $10 \mathrm{MHz}, 0.25^{\prime \prime}$ diameter, single element spherically focused (at $40 \mathrm{~mm}$ ) ultrasound transducer (type V312, Panametrics, Waltham, MA, USA). As illustrated in Figure 1, the transducer was mounted to a manual rotational device, such that the ultrasound beam intersected the axis of rotation at a distance of $D_{r o t}=58.53 \mathrm{~mm}$. The rotational device was in turn mounted to a $3 \mathrm{D}$ translation system. The $\mathrm{rf}$ signal from the pulser/receiver was digitized by a digital storage oscilloscope, DSO, (type 9450, LeCroy Corporation, Geneva, Switzerland), that in turn was connected via a GPIB interface to a Windows NT based workstation. By use of an RS232 interface, the 3D translation system could be controlled by this workstation as well. Custom designed software allowed control of the DSO and the 3D translation system directly from the data processing and visualization program MATLAB (The Mathworks Inc, Natick, MA, USA). The DSO control program allowed direct setup of all relevant instrument parameters. Vertical gain setting was automatically adjusted, to ensure maximum utility of the dynamic range of the analog-to-digital converter for each individual echo signal.

The ultrasound transducer was characterized using a $0.1 \mathrm{~mm}$ diameter glass sphere moulded into an agar block. 


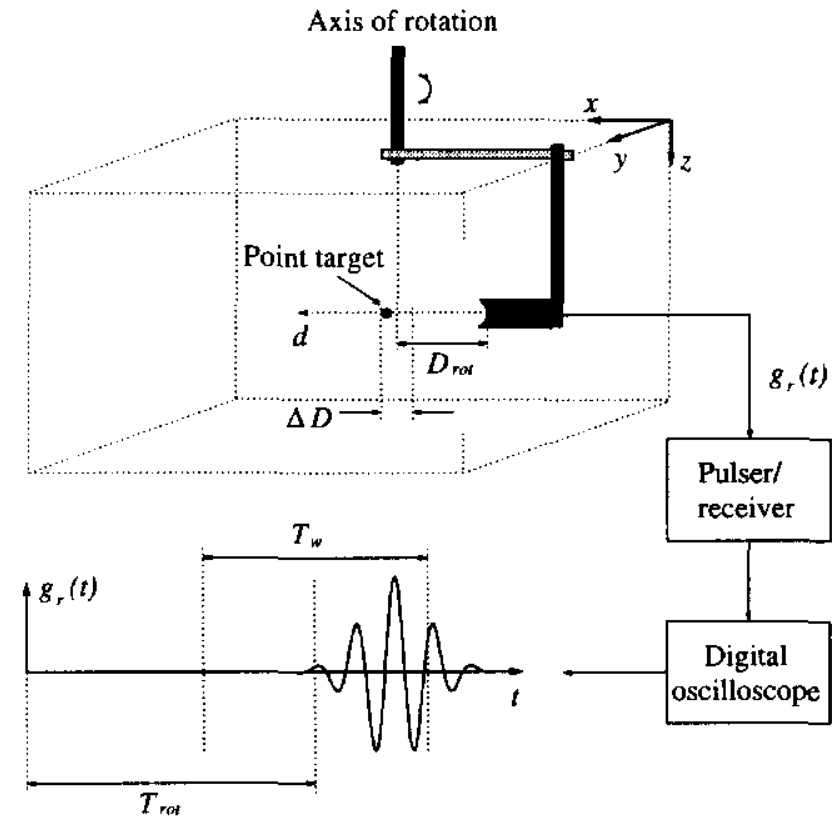

Figure 1 Single element spherically focused ultrasound transducer mounted to rotation axis via a horizontally mounted bar. The acoustic axis intersects the axis of rotation at an angle of $90^{\circ}$. The received signal (here illustrated for a single point scatterer located a little off the axis of rotation) was recorded in the interval specified by $T_{w}=\Delta D / c$.

At the range $D_{r o t}$ the $-3 \mathrm{~dB}$ lateral beam width was 1.4 $\mathrm{mm}$.

The precision of the combined rotation and translation system was measured with the same point target, and the maximal spatial uncertainty was found to be below 0.25 $\mathrm{mm}$.

\section{Clinical Procedure and Data Acquisition}

From December 1994 to April 1996 plaques from 13 patients with carotid artery disease (12 men and 1 woman, median age 63 years, range $54-73$ ) were removed by prophylactic carotid endarterectomy. All patients were referred to the Department of Vascular Surgery, Rigshospitalet, Copenhagen, with neurological symptoms from the same side as the stenotic carotid artery. The neurological symptoms were stroke $(n=3)$, transient ischemic attack (TIA) $(n=9)$ or amaurosis fugax $(n=1)$. All patients gave informed consent and the study protocol was approved by the medical ethics committee for Copenhagen and Frederiksberg counties (\#KF 01-375/94).

The plaques were fixed in formalin for a duration of up to 2 weeks before scanning. The longitudinally opened plaques were fixed to an acrylic frame in an "as open as possible" condition by means of four sutures, as shown in Figure 2. The frame with plaque was placed in a thin-walled latex bag with formalin, as the scanning could take op to 24 hours.

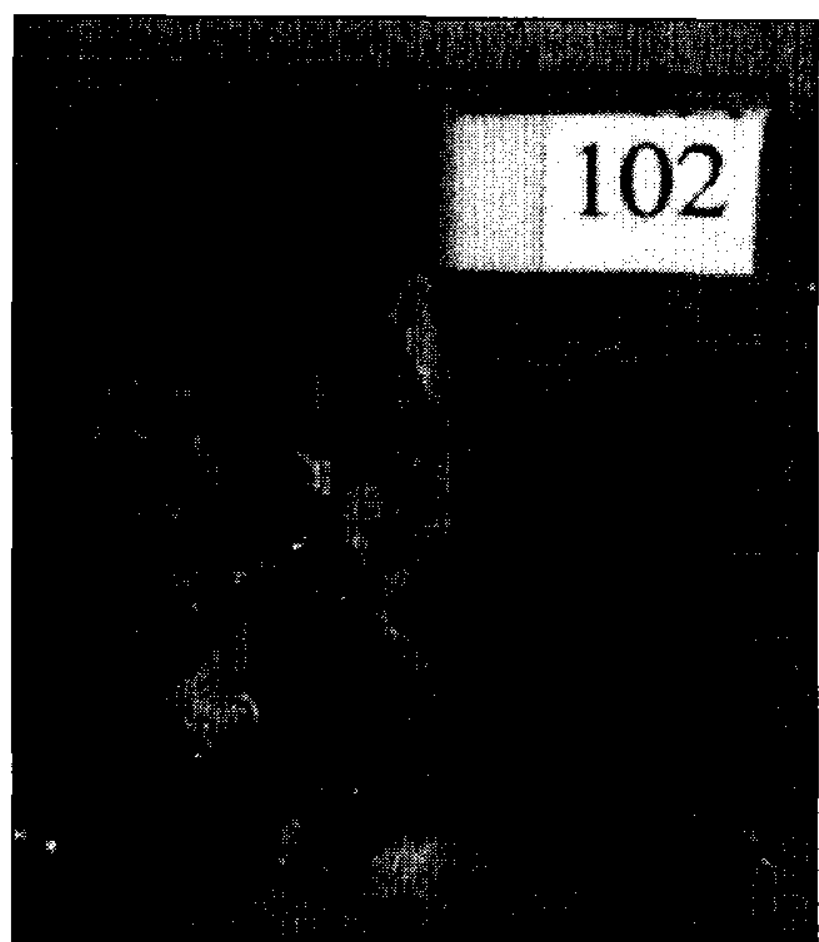

Figure 2 Picture of carotid plaque in vitro fixed with sutures mounted to an acrylic frame (not shown). The two axes have tick marks every $0.5 \mathrm{~cm}$.

The same part of the ultrasound beam was used for all range cells which required the transducer moved in 3D. The advantage of this very time consuming method was high precision in spatial definition of the range cell. The received signal segment, $g_{r}(x, y, z, \theta, t)$, for a given range cell, $(x, y, z)$ and angle, $\theta$, was extracted by windowing out a small segment from the entire received signal. This segment was located from $T_{\text {rot }}-T_{w} / 2$ to $T_{r o t}+T_{w} / 2$, where $T_{r o t}=2 D_{r o t} / \mathrm{c}$ and $T_{w}=\Delta D / c$ is the window length $(\Delta D \sim 4 \mathrm{~mm})$.

\section{Histological Analysis}

Following ultrasound scanning, sutures were removed from the plaques which were subsequently laced back into a shape that matched the in vivo form as well as possible. After measuring the length of the entire specimen, transverse blocks were cut at approximately $3 \mathrm{~mm}$ distance. After processing the blocks in paraffin, microtome sections were cut from each block. These were stained with Haematoxylin and Eosin as well as a connective tissue stain as Van Gieson and eventually stained for elastin. The histological analysis was performed by a single experienced pathologist using a microscope. The microscopic images were transferred to a computer for outlining and determination of area. The features analyzed were area of lipid, haemorrhage, thrombus, calcification and fibrous tissues. The volu- 
metric contents of the five materials in each plaque could then be estimated from the spatial separation of the microtome sections.

\section{Signal Processing and Feature Extraction}

In order to obtain range cells with an omnidirectional sensitivity function, the axial resolution size had to be made equal to the lateral resolution size. This was done by multiplying the received signal segment, $g_{r}(x, y, z, \theta$, $t)$, from each range cell with a Gaussian window

$g_{w}(t)=\exp \left[-\frac{2 \ln (2)}{T_{-3 d B}^{2}}\left(t-\frac{r_{r o t}}{2}\right)^{2}\right]$

The $-3 \mathrm{~dB}$ length of this window, $T_{-3 \mathrm{~dB}}$, roughly corresponded to the $-3 \mathrm{~dB}$ width of the ultrasound beam at $D_{r a r}$ The result of the multiplication was then integrated in order to measure the energy of the received signal from that range cell

$E(x, y, z, \theta)=\int_{-T_{w} / 2}^{T_{w} / 2}\left|g_{w}(t) g_{r}(x, y, z, \theta, t)\right|^{2} d t$

$E(x, y, z, \theta)$ thus formed a set of five volume images. Before the images could be used, the range cells containing sutures had to be masked out manually. Very simple features could then be extracted from these volume images. In Figure 3, a given range cell is considered, and it is indicated how the five associated voxel values can be used to calculate six simple features. For each plaque, a new volume image was found for each feature. In this paper, these "feature volume images" were reduced to single scalars by means of simple averaging. If $\Gamma$ is an operator that can take the form $\min \{\}, \operatorname{mean}\{\}$ and $\max \{\}$, then the six scalars, for each plaque, can be written as:

$$
F_{E, \Gamma}=\operatorname{mean}_{x, y, z}\{\Gamma\{E(x, y, z, \theta)\}\}
$$

where $\theta=-20^{\circ},-10^{\circ},-0^{\circ}, 10^{\circ}$ and $20^{\circ}$, and

$$
\begin{aligned}
F_{s, \Gamma} & =\operatorname{mean}_{x, y, z}\{\Gamma\{\mid E(x, y, z, \theta) \\
& -E(x, y, z, \theta+\Delta \theta) \mid\}\}
\end{aligned}
$$

where $\Delta \theta=10^{\circ}$ and $\theta=-20^{\circ},-10^{\circ},-0^{\circ}, 10^{\circ}$.

\section{Analysis Technique}

The correlation between the histological results, represented in vector $Y$, and an ultrasound feature, contained in matrix $\underline{X}$, were investigated using the linear model, $X=\underline{X \beta}$. The regression parameters, $\underline{\beta}$, were found based on the least squares solution. The residuals could be calculated as: $\underline{\varepsilon}=\underline{Y}-\underline{\underline{\beta}}$.
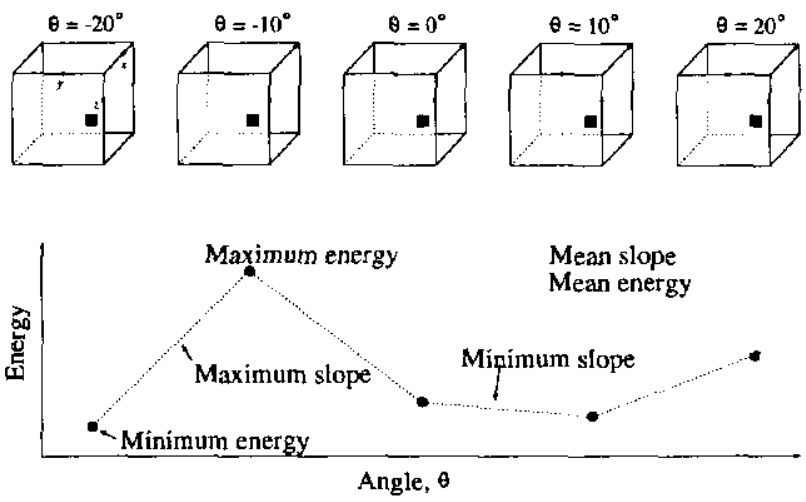

Figure 3 Upper panel: Illustration of volume images from five different angles. One voxel from each volume image is considered. These five values are the energy from the subvolume (range cell) as a function of angle. Lower panel: The five energy values plotted as a function of angle. Also shown are six different features calculated from the five energy values.

An efficient and correct way to search for, and identify features predicting the content of a given material is to investigate a large number of possible features on a subset of the data available (training set) and then test the performance of each feature on the remaining data (test set). This approach was not possible at the present stage of the project, where only 13 plaques had been scanned. Consequently, only the six above features were investigated for the entire data set, as these were all expected to correlate with the histological results, based on Picano's findings ${ }^{[6]}$.

\section{Results}

A total of 13 plaques have been scanned in vitro in 3D. Of these, the first 3 plaques were scanned from the insonification angles $-20^{\circ}, 0^{\circ}$ and $20^{\circ}$, while the remaining were scanned from the insonification angles $-20^{\circ},-10^{\circ}, 0^{\circ}, 10^{\circ}$ and $20^{\circ}$. The distance between the center of neighboring range cells was $1.4 \mathrm{~mm}$ and signals from approximately 1000 range cells were recorded for each plaque.

The relative content of fibrous tissues, calcification and "soft" materials (lipid materials, thrombosis and blood, which are assumed indistinguishable by ultrasound) were $72.5 \pm 9.9 \%, 0.7 \pm 0.5 \%$ and $26.9 \pm 10.1 \%$, respectively (mean \pm one standard deviation).

The correlation between ultrasound features and histological results was investigated for content of calcification and content of fibrous tissues. The best correlation was obtained with the latter. The results are depicted in Figure 4 which shows a scatter plot of $F_{S_{\text {min }}}$ versus relative content of fibrous tissues together with the estimated regression line. 


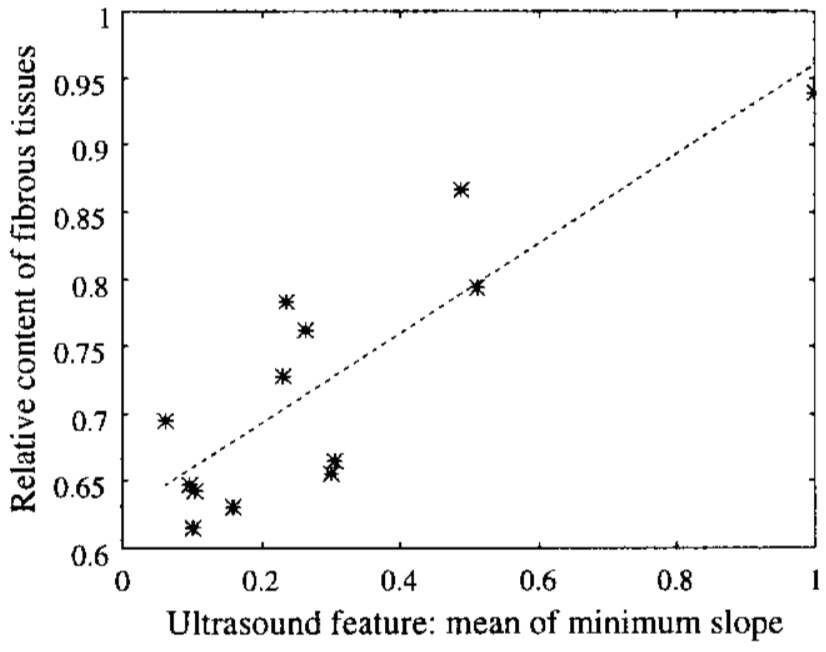

Figure 4 Correlation between $F_{S_{\text {min }}}$ and histologically estimated content of fibrous tissues. The parameters for the regression line is: $\alpha=0.62, \beta=0.33 . p<0.0002$ and the correlation coefficient is $r=0.86 . \overline{|\varepsilon|}=0.04$.

\section{Discussion}

The preliminary results in Figure 4 suggest that minimum slope of the received signal energy (spatially averaged over the entire plaque), $F_{S, \min }$, is proportional to relative content of fibrous tissues. The plaques investigated in this study consisted mainly of fibrous tissues and lipid materials. Therefore, these results could be compared to the findings of Picano et al ${ }^{[6]}$, for fatty, fibrofatty and fibrous plaques: For these three groups of plaques, they found that the received signal energy over a $60^{\circ}$ angular span varied increasingly. Specifically, while both fatty and fibrofatty plaques featured angular intervals with no angle-dependence, fibrous plaques displayed continuous angle-dependence. As $F_{S, \text { min }}$ is a measure of the magnitude of the slope, in the interval where the angle-dependence is smallest, the present results are in good agreement with the results of Picano et al.

It should also be noted that the preliminary results presented here are more encouraging than the results found in an investigation of clinical B-mode images ${ }^{[4]}$. This is not surprising, as the ultrasound data used in [4] were recorded in vivo and thus only originated from a single scan plane. Furthermore, the echo signals were disturbed by the inhomogeneous transmission medium between transducer and plaque. In addition, in studies like [4], the angle-dependence is a problem, not an extra source of information.

Despite the good preliminary results presented in this paper, a number of sources of error exist, which should be kept in mind when evaluating the performance of the method:

a) The plaque was only scanned over an angle interval of $40^{\circ}$ in one plane. Thus the entire angledependence pattern (a complete $180^{\circ}$ scanning in two planes) is not known, and only an estimate of the angle-dependence pattern can be obtained.

b) The ultrasound method used in this study might be much more accurate than the pathological analysis, which presently is considered the "golden standard". First, sections were cut with a coarse spatial sampling interval of $3 \mathrm{~mm}$. Second, the histological constituents were often mixed, making drawing of exact borders difficult. Thus the histological results might be associated with a relatively larger error compared to the ultrasound data.

\section{Conclusions}

Thirteen plaques, removed by carotid endarterectomy, have been scanned with ultrasound and data related to a subsequent histological analysis. The preliminary results indicate good correlation between an ultrasound feature representing angle-dependence and content of fibrous tissues $(p<0.0002)$.

\section{Acknowledgements}

CADUS is supported by the Danish Technical and Medical Research Councils. The authors gratefully acknowledge the help by M.S. Students Niels Nordmann in carrying out part of the measurements. The authors gratefully acknowledge the help of assoc. prof. $O$. T Andersen and M.Sc. S. K. Jespersen in proofreading the manuscript.

\section{References}

[1] Bock $R$, Gray-Weale A, Mock $P$ et al: The natural history of asymptomatic carotid artery disease. $J$. Vasc. Surg. 17, pp 160-171. 1993.

[2] Eliasziw $M$, Streifler $J Y$, Fox AJ, Hachinski VC, Ferguson $G G$ \& Barnett $H J M$ : Significance of Plaque Ulceration in Symptomatic Patients with High-Grade Carotid Stenosis. Stroke. pp. 304-308. 1994.

[3] European Carotid Surgery Trialist Group: MRC European carotid surgery trial: Interim results of symptomatic patients with severe $\mathbf{( 7 0 - 9 9 \% )}$ or with mild (0-29\%) stenosis. Lancet. 337. pp 1235-1243. 1991. [4] Jespersen SK, Grønholdt M-LM, Wilhjelm JE, Wiebe $B$, Hansen $L K$, Sillesen $H$ : Correlation between ultrasound $B$-mode images of carotid plaque and histological examination. Proceedings of the IEEE Ultrasonics Symposium, San Antonio, Texas, USA, Nov. 3-6. 1996. [5] North American Symptomatic Carotid Endarterectomy Trial Collaborators: Beneficial effect of carotid endarterectomy in symptomatic patients with highgrade carotid stenosis. New Engl. J. med. 325; pp. 445-453. 1991.

[6] Picano E, Landini L, Distante A, Salvadori $M$, Lattanzi $F$, Masini $M$ and $L$ 'Abbate $A$ : Angle dependence of ultrasonic backscatter in arterial tissues: a study in vitro. Circulation, vol. 72, no. 3, pp. 572-576. 1985. 